\title{
New expedited process to accelerate the pathogen identification and antimicrobial susceptibility tests
}

\author{
Jingan Lin ${ }^{1-3}$, Bin Yang ${ }^{1-3}$, Paul Okunieff ${ }^{4}$, Lurong Zhang ${ }^{1-4 *}$ and Jianhua Lin ${ }^{1-3 *}$ \\ ${ }^{1}$ First Affiliated Hospital of Fujian Medical University, Fuzhou, China \\ ${ }^{2}$ Fujian Key Lab of Individualized Active Immunotherapy, Fuzhou, China \\ ${ }^{3}$ Key Lab of Radiation Biology of Fujian Province Universities, Fuzhou, China \\ ${ }^{4}$ Department of Radiation Oncology, University of Florida, Gainesville, Florida 32610, USA
}

\begin{abstract}
Background: Sepsis requires an appropriate and quick treatment based on a fast determination of pathogen and antimicrobial susceptibility. It is much needed to explore a new protocol to shorten the turnaround time of currently standard process (SP) for pathogen identification (PI) and antimicrobial susceptibility tests (AST) used in most of hospitals without addition expensive devices.
\end{abstract}

Materials and methods: This study reported a new expedited process (NEP) that directly took 10 ml positive culture media from BD BACTEC bottles, lysed blood cells, obtained the bacterial pellet and directly processed for PI and AST. A side-by-side comparison between NEP and SP was carried out in 20 patients.

Results: While NEP shorten the turnaround time for at least 1 day, the side-by-side comparison showed: (a) NEP yielded the PI results identical to SP; (b) in 305 paired AST: 297 (97.38\%) were identical, only 4 (1.31\%) had major error (Resistant to Sensitive or Sensitive to Resistant) and 4 (1.31\%) had minor error (Intermediate to Sensitive or Resistant). Advantages of NEP are: (1) compared with currently used SP, NEP does not need overnight dish culture for colonies, saving manpower and materials, and yielding the reports of PI and AST at least 1day earlier than SP; (2) compared to other newly developed rapid-techniques, NEP utilizes SP devices without addition of new expensive devices and reagents, which is acceptable easily by all current SP users.

Conclusion: NEP is acceptable to replace SP with an advantage of shortening at least 1day turnaround time for PI and AST, which will have a significant impact on the treatment decision-making and outcome of patients with systemic infection.

\begin{abstract}
Abbreviations: SP: Standard Process; NEP: New Expedited Process; McF: Mcfarland turbidimetric unit (bacterial concentration); PI: Pathogen Identification; AST: Antimicrobial Susceptibility Tests

\section{Introduction}

Sepsis, caused by infections, trauma, major organ dysfunction and end-stage of many diseases, such as cancer, aging, etc., is the most critical situation that requires immediately appropriative treatment, since each hour delay will increase mortality rates dramatically, leading to up to $30 \%$ death [1-4]. Obviously, the early turnaround times for PI and AST are critical for rescuing life [5-8].
\end{abstract}

At present, in most of hospitals use the standard procedure (SP), which takes at least 2-3 days to report the pathogen stain and antimicrobial susceptibility [9-12]: on day one, to culture blood in BD BACTEC bottle; on day two, if culture is positive, the pathogen is inoculated to the dish for colonies to grow overnight; on day three, all grown colonies are collected, justified to concentration of $0.5 \mathrm{McF}$ for PI and AST using VITEK 2 (bioMerieux Inc) systems. In 2018, Tabak et al. [12] analyzed 165,593 blood specimens collected from 13 US hospitals in January 2015 to June 2016 and reported that overall average turnaround time from specimen collection for Gram-stain of positive culture, PI, and AST was approximately 1,2, and 3 days, respectively [12]. Within 2-3 days without information of PI and AST, physicians have to treat patients based on the clinical symptoms and their experience [1,9-15].
Great efforts have been made to develop various instrumentassisted PI and AST systems to shorten the turnaround time to in hours rather than SP in days [16-17]. Recently, the multiplex polymerase chain reaction-based rapid diagnostic tests, such as Film Array Blood Culture Identification (FA-BCID), is developed to detect pathogens and antimicrobial resistance genes for rapid reports in about one hour, however, an initial blood culture is still required for a positive microbial growth [18]. A matrix-assisted laser desorption/ionization time-offlight mass spectrometry (MALDI-TOF MS) is also used for a rapid report [19]. All these new techniques require expensive devices, which are not available in most of hospitals [12]. Therefore, it badly need to utilize SP currently used devices and systems by changing protocol to shorten the turnaround time of PI and AST before most of hospitals could be equipped with expensive FA-BCID or MALDI-TOF MS.

${ }^{\star}$ Correspondence to: Jianhua Lin, First Affiliated Hospital of Fujian Medical University, Fuzhou, China, E-mail: jianhual@126.com

Lurong Zhang, Fujian Key Lab of Individualized Active Immunotherapy and Key Lab of Radiation Biology of Fujian Province Universities, Fuzhou, China, E-mail: lurongzhang@ufl.edu / lz8506@163.com

Key words: pathogen identification, antimicrobial susceptibility, early diagnosis of sepsis

Received: October 20, 2018; Accepted: November 01, 2018; Published: November 05, 2018 
After carefully observation of the currently used SP, we found that the inoculation of bacteria from bottle to dish for further cultured overnight could be omitted, due to: (1) instead of picking up single colony, all the colonies grown overnight in the whole dish were harvested together, pooled into the same tube and adjusted to $0.5 \mathrm{McF}$ for PI and AST; (2) in almost all the cases, the pathogen report from VITEK 2 reader of whole dish is single bacterial strain; and (3) very few culture dish grows more than one strain of bacteria. Therefore, we believe that directly processing the bacterial positive media from the BD BACTEC bottle is likely to yield the same results of PI and AST as these from the re-culture in dish overnight. To prove if NEP omits the step of overnight dish culture could yield the same results as SP (Figure 1 ), we performed this study with a goal of shorten the turnaround time for at least one day without changing any used device and associated reagents, which could be welcome by physicians and patients for its aiding in the early and accurate treatment of sepsis.

\section{Materials and methods}

\section{Sample collection}

Patients with 2 of 4 following clinical symptoms were highly suspected as sepsis [16]: (1) a body temperature of $>38^{\circ} \mathrm{C}$ or $<36^{\circ} \mathrm{C}$; (2) a heart rate $>90$ beats per min; (3) a respiratory rate $>20$ breaths per minute or an arterial $\mathrm{CO}_{2}$ pressure of $<32 \mathrm{~mm} \mathrm{Hg}$; and (4) white blood cell counts of $>12,000$ cells/ L or $<4000$ cells/ L or $>10 \%$ immature forms. Then, their blood samples were collected and cultured in BD BACTEC bottle (Becton Dickinson and Company Sparks, MD, USA 21157) were performed for 7 day [17-18]. Twenty cultured blood samples out of about 200 samples from hospitalized patients in 4 months of year 2015 were positive for either gram-negative or grampositive bacteria in 12-72 hours after culture. The positive samples in BD BACTEC bottle were further processed according to procedures of either routine SP or our NEP. The sample collection was approved by the institutional review board of First Affiliated Hospital of Fujian Medical University (protocol\# 2015-084).

\section{Standard process (SP)}

As showed in figure 1, the positive samples from BD BACTEC bottle were inoculated in to bacterial culture dish for overnight culture. Next day (about 20-22 hours later), all colonies grown in the dish were

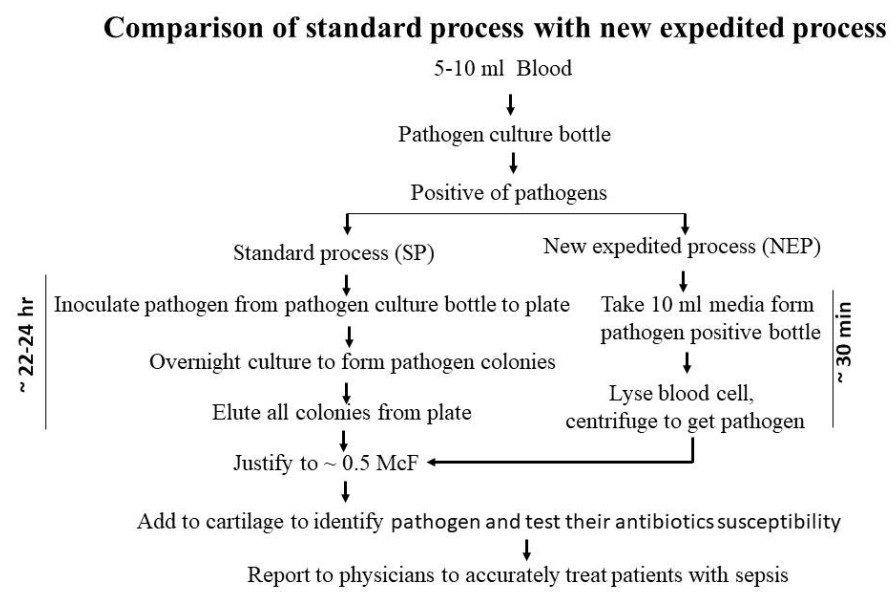

Figure 1. Comparison of processes between SP and NEP: Broth taken from a blood culture positive bottle, either inoculated on to dish for overnight culture of colonies for $\mathrm{SP}$ or transferred $10 \mathrm{ml}$ broth to a $50 \mathrm{ml}$ tube for lysis of blood cellular portion for NEP. followed by making $0.5 \mathrm{McF}$ for PI and AST with VITEK 2 device and its associated cards. NEP omitted the dish re-culture step, which yielded results about 22-24 h earlier than SP. eluted with saline and justified to the final bacterial concentration of $0.5 \mathrm{McF}$ with Mcfarland turbidimetric device (DensiCHEK plus, Biomerieux, Andover, Massachusetts, USA), then used VITEK 2 system and its cards for PI \{Gram-Negative (or Positive) Identification cards\} and AST \{Gram-Negative (or Positive) AST cards\}.

\section{New expedited process (NEP)}

As showed in figure $1,10 \mathrm{ml}$ bacterial positive culture media from BD BACTEC bottle was taken out with a $20 \mathrm{ml}$ of sterilized syringe, placed in $50 \mathrm{ml}$ sterilized tube, added $35 \mathrm{ml}$ of sterile water and then 5 $\mathrm{ml}$ of $10 \times$ filtered blood cell lysis buffer $(\mathrm{NH} 4 \mathrm{Cl} 83 \mathrm{~g}$; $\mathrm{NaHCO} 310 \mathrm{~g}$; EDTA $370 \mathrm{mg}$; addition of sterile water to $500 \mathrm{ml}$ ), sharking the tube gently by a rotator ( $60 \mathrm{rpn}$ ) for about $8-10 \mathrm{~min}$ until the solution in the tube was completely transparent, then centrifuged ( $1500 \mathrm{~g}, 10 \mathrm{~min}$ ) to obtain the bacterial pellet. After wash with saline once, the bacterial pellet was re-suspend in saline and justified to $0.5 \mathrm{McF}$ followed by using VITEK 2 devices and cards for PI and AST as done in SP above.

\section{Pathogen identification}

The same volume $(0.5 \mathrm{ml})$ of $0.5 \mathrm{McF}$ each from SP (colonies harvested from overnight culture dish) and NEP (10 $\mathrm{ml}$ broth directly taken from positive BD BACTEC bottle, lysis of blood cell for pellet of bacteria) from each of 20 patients was transferred separately into Gram-Negative (or Positive) Identification cards specially made by bioMerieux Inc and inserted into VITEK 2 device (bioMerieux Inc, 595 Anglum Road, Hazelwood, MO 63042 (USA) for PI. The results of bacterial strains were compared side-by-side between SP and NEP.

\section{Antimicrobial susceptibility tests (AST)}

According to the result of Gram stain, the same volume $(0.5 \mathrm{ml})$ of $0.5 \mathrm{McF}$ each from SP and NEP bacterial preparation of same patient was also transferred separately into either Gram-positive test kit (VITEK 2AST-GP67, including 21 drug tests) or Gram-negative test kit (VITEK 2AST-GN09, including 22 drug tests) and then inserted into VITEK 2 device for the AST. The results of AST were compared side-by-side between SP and NEP.

\section{Statistical analysis}

The McNemar-bowker paired chi-square test was used to test the differences between the two procedures used in the same sample, $\mathrm{X}^{2}$ $=2.000, p<0.05$ was statistically significant difference. The kappa consistency test was used to examine the consistency of the two procedures. When Kappa was $>0.9$, indicating that the consistency was excellent.

\section{Results}

\section{The pathogen identification is identical between SP and NEP}

As shown in figure $2 \mathrm{~A}$, in 20 blood culture positive cases, media from each positive culture bottle was taken either inoculating into culture dish for isolates as SP or $10 \mathrm{ml}$ subjected to NEP. The PI results from two procedures was identical, i.e. $100 \%$ category agreement in all pathogen systemically invaded in 20 patients, including Staphylococcus aureus $(\mathrm{n}=7)$, Klebsiella pneumoniae ssp pneumoni $(\mathrm{n}=6)$, Escherichia coli $(\mathrm{n}=3)$, Acinetobacter baumannii $(\mathrm{n}=1)$, Enterococcus faecium ( $\mathrm{n}$ $=1)$, Pseudomonas aeruginosa $(\mathrm{n}=1)$, Enterobacter aerogenes $(\mathrm{n}=1)$. Data demonstrated that: (1) NEP yielded the PI not only identical to SP, but also about 24 hours earlier than SP; and (2) the invaded genus was monomicrobial in all these 20 cases. 


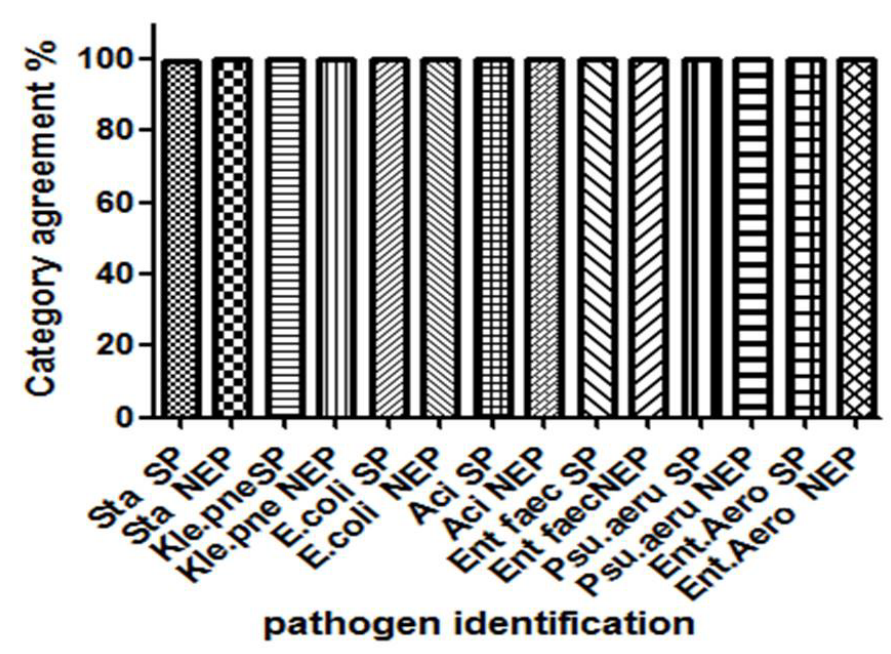

Figure 2A. The pathogen identification is identical between SP and NEP: In 20 patients tested for genus, SP and NEP yielded identical pathogens (Mcnemar-bowker paired chisquare test, $P>0.5$ ): 7 cases with Staphylococcus aureus, 6 Klebsiella pneumoniae, 3 Escherichia coli, 1 Acinetobacter baumannii, 1 Enterococcus faecium, 1 Pseudomonas aeruginosa and 1 Enterobacter aerogenes. All 20 patients were systemically infected with mono-microbial.

\section{The results of AST are largely agreed between SP and NEP}

The AST includes the following antibiotics: gentamicin (GM), levofloxacin (LEV), aztreonam (ATM), piperacillin (PIP), nitrofurantoin (FT), trimethoprim/sulfa (SXT), erythroprim/sulfa (E), ciprofloxacin (CIP), amikacin (AN), imipenem (IPM), clindamycin (CM), quinupristin/dalfopristin (QDA), moxifloxacin (MXF), tetracycline (TE), vancomycin (VA), ampicillin (AM), ampicillinsulbactam (SAM), tobramycin (TM), cefotetan (CTT), ceftazidime (CAZ), ceftriaxone (CRO), aztreonam cefepime (FEP), piperacillin (TZP), piperacillin (PIP), tigecycline (TGC), oxacillin (OX1), cefuroxime (CXM), linezolid (LNZ), penicillin-g/ benzylpenicillin (P), rifampin (RA), and meropenem (MEM).

Compared SP with NEP, as shown in figure $2 \mathrm{~B}$, among total 305 paired AST (SP v.s. NEP), 297 paired results (297/305, 97.38\%) were identical. There were 4 tests $(4 / 305,1.31 \%)$ with minor errors, sensitivity from intermediate to sensitive or resistant (I to S or I to R), shown 1 with Staphylococcus aureus (Table 1), 1 with Escherichia coli (Table 3), 2 with Enterobacter aerogenes (Table 7). There were 4 tests $(4 / 305,1.31 \%)$ with major errors, such as sensitivity from resistant or sensitive (R or S) to sensitive or resistant ( $\mathrm{S}$ or R), shown 3 with Staphylococcus aureus (Table 1), and 1 with Pseudomonas aeruginosa (Table 6).

Tables 1-7 demonstrated the detail of each paired AST with the names of antibiotics. The AST agreement rate in total 305 tests was $97.38 \%$ with $4(1.31 \%)$ minor errors (I to $\mathrm{S}$ or $\mathrm{R}$ ) and 4 (1.31\%) major errors (S to $\mathrm{R}$ or $\mathrm{R}$ to $\mathrm{S}$ ). Specifically, as shown in figure $2 \mathrm{C}$, among 31 antibiotics tested in 305 paired samples, CA, RA, GM and CAZ each had one major error, PIP had two minor errors, LNZ and IPM each had one minor error. The data suggest that NEP yields very low error rate, therefore, it is acceptable as compared to SP with advantage of one day earlier reports of AST to physicians.

\section{Discussion}

In this study, we proved that our NEP has the following advantages: (1) shortening for about one day in the turnaround time of PI and AST, which is critical for appropriate treatment and rescue the life of patients with sepsis; (2) compared with the SP, our NEP yielded identical result in PI and $97.38 \%$ agreement in AST, indicating that NEP is acceptable without lowing the laboratory test standard; (3) omitted one step of the inoculation of bacteria from bottle to dish, which not only advancing the whole process for about one day, but also help in saving lab manpower and materials, which is benefit to laboratory staff and patients; (4) due to only omitted one step without changing any other process and devices/reagents used, it should be easy to apply to hospital using their currently used device, such as the VITEK 2 system (bioMerieux Inc) for PI and AST.

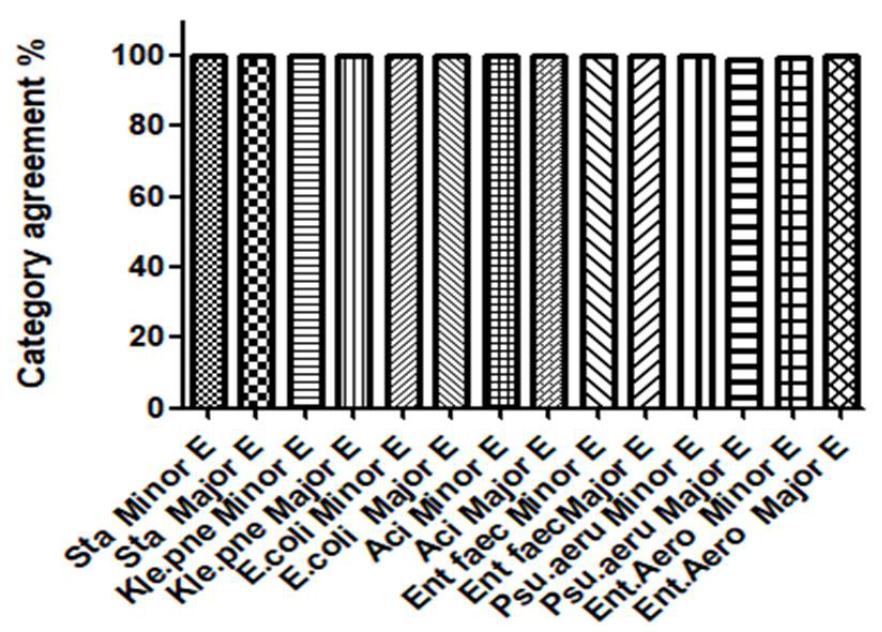

AST error types in different pathogens tested

Figure 2B. The antimicrobial susceptibility is largely agreed between SP and NEP Totally, 305AST were performed in 20 cases with 7 pathogens. 297 out of $305(97.38 \%)$ AST results were identical, 4 with minor error $(1.31 \%)$ and 4 with major error $(1.31 \%$, the kappa consistency test, $P>0.9$ ).

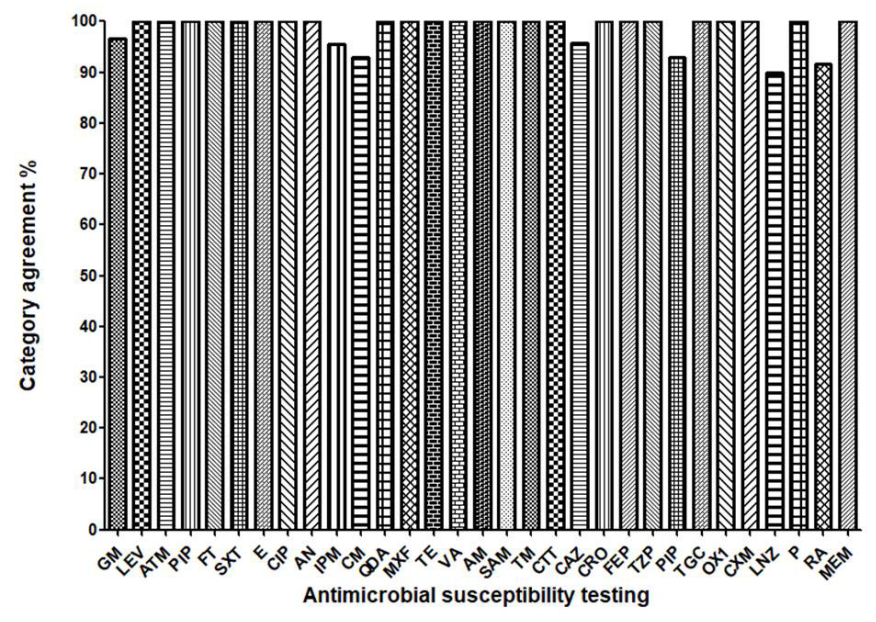

Figure 2C. The antibiotic sensitivity agreement between SP and NEP: A panel of antibiotics indicated in VITEK 2AST-GP67 and VITEK 2AST-GN09 cards were used in AST , including Gentamicin (GM), Levofloxacin (LEV), Aztreonam (ATM), Piperacillin (PIP), Nitrofurantoin (FT), Trimethoprim/sulfa (SXT), Erythroprim/sulfa (E), Ciprofloxacin (CIP), Amikacin (AN), Imipenem (IPM), Clindamycin (CM), Quinupristin/dalfopristin (QDA), Moxifloxacin (MXF), Tetracycline (TE), Vancomycin (VA), Ampicillin (AM), Ampicillin-sulbactam (SAM), Tobramycin(TM), Cefotetan (CTT), Ceftazidime (CAZ) Ceftriaxone (CRO), Aztreonam cefepime (FEP), Piperacillin (TZP), Piperacillin (PIP) Tigecycline (TGC), Oxacillin (OX1), Cefuroxime (CXM), Linezolid (LNZ), Penicillin-G/ Benzylpenicillin (P), Rifampin (RA), Meropenem (MEM), etc. The AST results of SP and NEP had a $97.38 \%$ agreement rate. 4 (1.31\%) minor errors were with linezolid (LNZ), Piperacillin (PIP) and Imipenem (IPM), respectively; and 4 (1.31\%) major errors were with clindamycin (CM), Rifampin (RA), Gentamicin (GM) and Ceftazidime (CAZ), respectively (the kappa consistency test, $p>0.9$ ). 
Table 1. Antibiotics susceptibility tests for Staphylococcus with two procedures*

\begin{tabular}{|c|c|c|c|c|c|c|c|c|c|c|c|c|c|c|}
\hline & \multicolumn{2}{|c|}{ Case 1} & \multicolumn{2}{|c|}{ Case 2} & \multicolumn{2}{|c|}{ Case 3} & \multicolumn{2}{|c|}{ Case 4} & \multicolumn{2}{|c|}{ Case 5} & \multicolumn{2}{|c|}{ Case 6} & \multicolumn{2}{|c|}{ Case 7} \\
\hline & SP & NEP & SP & NEP & SP & NEP & SP & NEP & SP & NEP & SP & NEP & SP & NEP \\
\hline Pathogen identification & Sta. homi & Sta.homi & Sta.aure & Sta.aure & Sta.aure & Sta.aure & Sta.aure & Sta.aure & Sta..aure & Sta..aure & Sta..aure & Sta..aure & Staphylo & Staphylo \\
\hline Oxacillin & $\mathrm{S}$ & $\mathrm{S}$ & $\mathrm{R}$ & $\mathrm{R}$ & $\mathrm{R}$ & $\mathrm{R}$ & $\mathrm{S}$ & $\mathrm{S}$ & $\mathrm{S}$ & $\mathrm{S}$ & & & $\mathrm{R}$ & $\mathrm{R}$ \\
\hline Nitrofurantoin & $\mathrm{S}$ & $\mathrm{S}$ & $\mathrm{S}$ & $\mathrm{S}$ & & & & & S & $\mathrm{S}$ & & & $\mathrm{R}$ & $\mathrm{R}$ \\
\hline Trimethoprim/ sulfa & $\mathrm{S}$ & $\mathrm{S}$ & $\mathrm{R}$ & $\mathrm{R}$ & & & & & $\mathrm{S}$ & $\mathrm{S}$ & & & $\mathrm{R}$ & $\mathrm{R}$ \\
\hline Erythroprim/sulfa & & & & & $\mathrm{R}$ & $\mathrm{R}$ & $\mathrm{S}$ & $\mathrm{S}$ & $\mathrm{S}$ & $\mathrm{S}$ & & & $\mathrm{R}$ & $\mathrm{R}$ \\
\hline Ciprofloxacin & $\mathrm{R}$ & $\mathrm{R}$ & $\mathrm{R}$ & $\mathrm{R}$ & & & S & $\mathrm{S}$ & S & $\mathrm{S}$ & $\mathrm{S}$ & S & $\mathrm{S}$ & $\mathrm{S}$ \\
\hline Clindamycin & $\mathrm{S}$ & $\mathrm{S}$ & $\mathrm{R}$ & $\mathrm{R}$ & $\mathbf{S}$ & $\mathbf{R}$ & $\mathrm{S}$ & $\mathrm{S}$ & $\mathrm{S}$ & $\mathrm{S}$ & $\mathrm{S}$ & $\mathrm{S}$ & $\mathrm{S}$ & $\mathrm{S}$ \\
\hline Quinupristin/dalfopristin & $\mathrm{R}$ & $\mathrm{R}$ & $\mathrm{R}$ & $\mathrm{R}$ & & & $\mathrm{S}$ & $\mathrm{S}$ & $\mathrm{S}$ & $\mathrm{S}$ & $\mathrm{S}$ & $\mathrm{S}$ & & \\
\hline Rifampin & $\mathrm{S}$ & $\mathrm{S}$ & $\mathbf{R}$ & $\mathbf{S}$ & & & $\mathrm{S}$ & $\mathrm{S}$ & $\mathrm{S}$ & $\mathrm{S}$ & $\mathrm{S}$ & $S$ & $\mathrm{R}$ & $\mathrm{R}$ \\
\hline Moxifloxacin & $\mathrm{S}$ & $\mathrm{S}$ & & & & & $\mathrm{S}$ & $\mathrm{S}$ & $\mathrm{S}$ & $\mathrm{S}$ & $\mathrm{S}$ & $\mathrm{S}$ & $\mathrm{S}$ & $\mathrm{S}$ \\
\hline Penicillin-G & & & & & & & & & & & & & $\mathrm{S}$ & $\mathrm{S}$ \\
\hline Gentamicin & $\mathrm{S}$ & $\mathrm{S}$ & $\mathbf{S}$ & $\mathbf{R}$ & & & $\mathrm{S}$ & $\mathrm{S}$ & $\mathrm{S}$ & $\mathrm{S}$ & $\mathrm{S}$ & $\mathrm{S}$ & $\mathrm{S}$ & $\mathrm{S}$ \\
\hline Tetracycline & & & & & & & $\mathrm{S}$ & $\mathrm{S}$ & $\mathrm{S}$ & $\mathrm{S}$ & $\mathrm{S}$ & $\mathrm{S}$ & I & I \\
\hline Tigecycline & $\mathrm{S}$ & $\mathrm{S}$ & $\mathrm{S}$ & $\mathrm{S}$ & & & $\mathrm{S}$ & $\mathrm{S}$ & $\mathrm{S}$ & $\mathrm{S}$ & $\mathrm{S}$ & $\mathrm{S}$ & $\mathrm{R}$ & $\mathrm{R}$ \\
\hline Vancomycin & $\mathrm{R}$ & $\mathrm{R}$ & $\mathrm{S}$ & $\mathrm{S}$ & & & $\mathrm{S}$ & $\mathrm{S}$ & & & $\mathrm{S}$ & $\mathrm{S}$ & $\mathrm{S}$ & $\mathrm{S}$ \\
\hline Levofloxacin & $\mathrm{S}$ & $\mathrm{S}$ & $\mathrm{S}$ & $\mathrm{S}$ & $\mathrm{S}$ & $\mathrm{S}$ & $\mathrm{S}$ & $\mathrm{S}$ & $\mathrm{S}$ & $\mathrm{S}$ & $\mathrm{S}$ & $\mathrm{S}$ & $\mathrm{S}$ & $\mathrm{S}$ \\
\hline Benzylpenicillin & $\mathrm{S}$ & $\mathrm{S}$ & $\mathrm{S}$ & $\mathrm{S}$ & & & $\mathrm{R}$ & $\mathrm{R}$ & & & $\mathrm{S}$ & $\mathrm{S}$ & $\mathrm{S}$ & $\mathrm{S}$ \\
\hline Linezolid & S & $\mathrm{S}$ & I & $\mathrm{S}$ & & & $\mathrm{S}$ & $\mathrm{S}$ & & & $\mathrm{S}$ & $\mathrm{S}$ & & \\
\hline Nitrofurantoin & & & & & & & $\mathrm{S}$ & $\mathrm{S}$ & & & $\mathrm{S}$ & $\mathrm{S}$ & & \\
\hline Trimethoprim/sulfa & $\mathrm{S}$ & $\mathrm{S}$ & & & & & & & & & & & & \\
\hline
\end{tabular}

S: Sensitive; R: Resistant; I: Intermediate; $*$ The kappa consistency Test was used, Kappa value $=0.875,95 \%$ CI: $0.743-0.972$, Kappa $>0.9$, indicating that the consistency was excellent.

Table 2. Antibiotics susceptibility tests for six cases of Klebsiella pneumoniae ssp pneumoni with two procedures*

\begin{tabular}{|c|c|c|c|c|c|c|c|c|c|c|c|c|}
\hline & \multicolumn{2}{|c|}{ Case 8} & \multicolumn{2}{|c|}{ Case 9} & \multicolumn{2}{|c|}{ Case 10} & \multicolumn{2}{|c|}{ Case 11} & \multicolumn{2}{|c|}{ Case 12} & \multicolumn{2}{|c|}{ Case 13} \\
\hline & SP & NEP & SP & NEP & SP & NEP & SP & NEP & SP & NEP & SP & NEP \\
\hline $\begin{array}{l}\text { Pathogen } \\
\text { identification }\end{array}$ & $\begin{array}{c}\text { Kleb. } \\
\text { pneumoni }\end{array}$ & $\begin{array}{c}\text { Kleb. } \\
\text { pneumoni }\end{array}$ & $\begin{array}{c}\text { Kleb. } \\
\text { pneumoni }\end{array}$ & $\begin{array}{c}\text { Kleb. } \\
\text { pneumoni }\end{array}$ & $\begin{array}{c}\text { Kleb. } \\
\text { pneumoni }\end{array}$ & $\begin{array}{c}\text { Kleb. } \\
\text { pneumoni }\end{array}$ & $\begin{array}{c}\text { Kleb. } \\
\text { pneumoni }\end{array}$ & $\begin{array}{c}\text { Kleb. } \\
\text { pneumoni }\end{array}$ & $\begin{array}{c}\text { Kleb. } \\
\text { pneumoni }\end{array}$ & $\begin{array}{c}\text { Kleb. } \\
\text { pneumoni }\end{array}$ & $\begin{array}{c}\text { Kleb. } \\
\text { pneumoni }\end{array}$ & $\begin{array}{c}\text { Kleb. } \\
\text { pneumoni }\end{array}$ \\
\hline Meropenem & S & $\mathrm{S}$ & S & $\mathrm{S}$ & $\mathrm{S}$ & $\mathrm{S}$ & $\mathrm{R}$ & $\mathrm{R}$ & $\mathrm{S}$ & $\mathrm{S}$ & $\mathrm{S}$ & $\mathrm{S}$ \\
\hline Piperacillin & $\mathrm{R}$ & $\mathrm{R}$ & $\mathrm{R}$ & $\mathrm{R}$ & $\mathrm{R}$ & $\mathrm{R}$ & $\mathrm{R}$ & $\mathrm{R}$ & $\mathrm{R}$ & $\mathrm{R}$ & $\mathrm{R}$ & $\mathrm{R}$ \\
\hline $\begin{array}{l}\text { Piperacillin/ } \\
\text { Tazobactam }\end{array}$ & $\mathrm{S}$ & S & $\mathrm{S}$ & S & $\mathrm{S}$ & S & $\mathrm{R}$ & $\mathrm{R}$ & S & S & S & S \\
\hline Amikacin & S & $\mathrm{S}$ & $\mathrm{S}$ & $\mathrm{S}$ & $\mathrm{R}$ & $\mathrm{R}$ & $\mathrm{R}$ & $\mathrm{R}$ & $\mathrm{S}$ & $\mathrm{S}$ & $\mathrm{S}$ & $\mathrm{S}$ \\
\hline Ampicillin & $\mathrm{R}$ & $\mathrm{R}$ & $\mathrm{R}$ & $\mathrm{R}$ & $\mathrm{S}$ & $\mathrm{S}$ & $\mathrm{R}$ & $\mathrm{R}$ & $\mathrm{R}$ & $\mathrm{R}$ & $\mathrm{R}$ & $\mathrm{R}$ \\
\hline $\begin{array}{l}\text { Ampicillin/ } \\
\text { Sulbactam }\end{array}$ & S & S & S & S & $\mathrm{S}$ & S & $\mathrm{R}$ & $\mathrm{R}$ & S & S & $\mathrm{R}$ & $\mathrm{R}$ \\
\hline Aztreonam & $\mathrm{S}$ & $\mathrm{S}$ & $\mathrm{S}$ & $\mathrm{S}$ & $\mathrm{S}$ & $\mathrm{S}$ & $\mathrm{R}$ & $\mathrm{R}$ & $\mathrm{S}$ & $\mathrm{S}$ & & \\
\hline Cefepime & S & S & S & $\mathrm{S}$ & $\mathrm{S}$ & $\mathrm{S}$ & $\mathrm{R}$ & $\mathrm{R}$ & & & S & $\mathrm{S}$ \\
\hline Cefuroxime & S & S & S & S & $\mathrm{S}$ & S & $\mathrm{R}$ & & $\mathrm{S}$ & S & S & $\mathrm{S}$ \\
\hline Cefuroxime Axetil & $\mathrm{S}$ & $\mathrm{S}$ & & & $\mathrm{S}$ & $\mathrm{S}$ & $\mathrm{R}$ & $\mathrm{R}$ & $\mathrm{S}$ & $\mathrm{S}$ & $\mathrm{R}$ & $\mathrm{R}$ \\
\hline Ceftriaxone & $\mathrm{S}$ & $S$ & $\mathrm{~S}$ & $\mathrm{~S}$ & $\mathrm{~S}$ & $\mathrm{~S}$ & $\mathrm{R}$ & $\mathrm{R}$ & $\mathrm{S}$ & $\mathrm{S}$ & $\mathrm{R}$ & $\mathrm{R}$ \\
\hline Ceftazidime & $\mathrm{S}$ & $\mathrm{S}$ & $\mathrm{S}$ & $\mathrm{S}$ & $\mathrm{S}$ & $\mathrm{S}$ & $\mathrm{R}$ & $\mathrm{R}$ & $S$ & $S$ & $\mathrm{R}$ & $\mathrm{R}$ \\
\hline Cefotetan & S & S & S & $\mathrm{S}$ & $\mathrm{S}$ & $\mathrm{S}$ & $\mathrm{R}$ & $\mathrm{R}$ & $\mathrm{S}$ & S & $\mathrm{S}$ & $\mathrm{S}$ \\
\hline Tobramycin & $\mathrm{S}$ & $\mathrm{S}$ & $\mathrm{S}$ & $\mathrm{S}$ & $\mathrm{S}$ & $\mathrm{S}$ & $\mathrm{R}$ & $\mathrm{R}$ & $\mathrm{S}$ & $\mathrm{S}$ & $\mathrm{S}$ & $\mathrm{S}$ \\
\hline Imipenem & $\mathrm{S}$ & $\mathrm{S}$ & $\mathrm{S}$ & $\mathrm{S}$ & $\mathrm{S}$ & $\mathrm{S}$ & $\mathrm{R}$ & $\mathrm{R}$ & $\mathrm{S}$ & $\mathrm{S}$ & $\mathrm{S}$ & $\mathrm{S}$ \\
\hline Levofloxacin & $\mathrm{S}$ & $\mathrm{S}$ & $\mathrm{S}$ & $\mathrm{S}$ & $\mathrm{I}$ & I & & & $\mathrm{S}$ & $\mathrm{S}$ & $\mathrm{S}$ & $\mathrm{S}$ \\
\hline Nitrofurantoin & $\mathrm{S}$ & $\mathrm{S}$ & I & I & $\mathrm{S}$ & $\mathrm{S}$ & & & $\mathrm{S}$ & $\mathrm{S}$ & $\mathrm{S}$ & $\mathrm{S}$ \\
\hline Trimethoprim/sulfa & $\mathrm{S}$ & $\mathrm{S}$ & $\mathrm{S}$ & $S$ & $\mathrm{~S}$ & $\mathrm{~S}$ & $\mathrm{R}$ & $\mathrm{R}$ & I & I & I & $\mathrm{R}$ \\
\hline Ciprofloxacin & $\mathrm{S}$ & $\mathrm{S}$ & & & & & $\mathrm{R}$ & $\mathrm{R}$ & & & $\mathrm{R}$ & $\mathrm{R}$ \\
\hline Gentamicin & $\mathrm{S}$ & $\mathrm{S}$ & & & & & $\mathrm{R}$ & $\mathrm{R}$ & S & S & $\mathrm{S}$ & $\mathrm{S}$ \\
\hline Cefuroxime & & & S & $\mathrm{S}$ & $\mathrm{S}$ & $\mathrm{S}$ & & & & & & \\
\hline
\end{tabular}

S: Sensitive; R: Resistant; I: Intermediate; Kleb. pneumoni: Klebsiella pneumoniae ssp pneumoni; *The kappa consistency Test was used, Kappa $=1.000$, indicating that the consistency was excellent. 
Lin J (2018) New expedited process to accelerate the pathogen identification and antimicrobial susceptibility tests

Table 3. Antibiotics susceptibility tests for Escherichia coli with two procedures*

\begin{tabular}{|c|c|c|c|c|c|c|}
\hline & Case 14 & & Case 15 & & Case 16 & \\
\hline & SP & NEP & SP & NEP & SP & NEP \\
\hline Pathogen identification & E.coli & E.coli & E.coli & E.coli & E.coli & E.coli \\
\hline Amikacin & $\mathrm{S}$ & $\mathrm{S}$ & $\mathrm{S}$ & $\mathrm{S}$ & $\mathrm{S}$ & $\mathrm{S}$ \\
\hline Ampicillin & $\mathrm{S}$ & $\mathrm{S}$ & $\mathrm{R}$ & $\mathrm{R}$ & $\mathrm{S}$ & S \\
\hline Ampicillin/ Sulbactam & $\mathrm{S}$ & $\mathrm{S}$ & $\mathrm{R}$ & $\mathrm{R}$ & $\mathrm{S}$ & $\mathrm{S}$ \\
\hline Aztreonam & $\mathrm{S}$ & $\mathrm{S}$ & $\mathrm{R}$ & $\mathrm{R}$ & $\mathrm{S}$ & $\mathrm{S}$ \\
\hline Nitrofurantoin & & & I & I & $\mathrm{S}$ & $\mathrm{S}$ \\
\hline Trimethoprim/ sulfa & $\mathrm{S}$ & $\mathrm{S}$ & $\mathrm{R}$ & $\mathrm{R}$ & $\mathrm{S}$ & $\mathrm{S}$ \\
\hline Ciprofloxacin & $\mathrm{S}$ & $\mathrm{S}$ & $\mathrm{R}$ & $\mathrm{R}$ & $\mathrm{S}$ & $\mathrm{S}$ \\
\hline Meropenem & $\mathrm{S}$ & $\mathrm{S}$ & $\mathrm{S}$ & $\mathrm{S}$ & $\mathrm{S}$ & $\mathrm{S}$ \\
\hline Piperacillin & $\mathrm{S}$ & $\mathrm{S}$ & $\mathrm{R}$ & $\mathrm{R}$ & $\mathrm{S}$ & $\mathrm{S}$ \\
\hline Piperacillin/ Tazobactam & $\mathrm{S}$ & $\mathrm{S}$ & & & $\mathrm{S}$ & I \\
\hline Gentamicin & $\mathrm{S}$ & $\mathrm{S}$ & $\mathrm{R}$ & $\mathrm{R}$ & $\mathrm{S}$ & $\mathrm{S}$ \\
\hline Cefepime & $\mathrm{S}$ & $\mathrm{S}$ & $\mathrm{S}$ & $\mathrm{S}$ & $\mathrm{S}$ & $\mathrm{S}$ \\
\hline Cefuroxime & $\mathrm{S}$ & $\mathrm{S}$ & $\mathrm{R}$ & $\mathrm{R}$ & $\mathrm{S}$ & $\mathrm{S}$ \\
\hline Cefuroxime Axetil & $\mathrm{S}$ & $\mathrm{S}$ & $\mathrm{R}$ & $\mathrm{R}$ & $\mathrm{S}$ & $\mathrm{S}$ \\
\hline Ceftriaxone & $\mathrm{S}$ & $\mathrm{S}$ & $\mathrm{R}$ & $\mathrm{R}$ & $\mathrm{S}$ & $\mathrm{S}$ \\
\hline Ceftazidime & $\mathrm{S}$ & $\mathrm{S}$ & $\mathrm{R}$ & $\mathrm{R}$ & $\mathrm{S}$ & $\mathrm{S}$ \\
\hline Cefotetan & $\mathrm{S}$ & $\mathrm{S}$ & $\mathrm{S}$ & $\mathrm{S}$ & $\mathrm{S}$ & $\mathrm{S}$ \\
\hline Tobramycin & $\mathrm{S}$ & $\mathrm{S}$ & $\mathrm{R}$ & $\mathrm{R}$ & $\mathrm{S}$ & $\mathrm{S}$ \\
\hline Imipenem & $\mathrm{S}$ & $\mathrm{S}$ & $\mathrm{S}$ & $\mathrm{S}$ & $\mathrm{S}$ & $\mathrm{S}$ \\
\hline Levofloxacin & S & $\mathrm{S}$ & $\mathrm{R}$ & $\mathrm{R}$ & S & S \\
\hline
\end{tabular}

E.coli: Escherichia coli; S: Sensitive; R: Resistant; I: Intermediate; ${ }^{*}$ The kappa consistency Test was used, Kappa value $=0.955,95 \%$ CI: $0.852-1.000$, Kappa $>0.9$, indicating that the consistency was excellent.

Table 4. Antibiotics susceptibility tests for Acinetobacter baumannii with two procedures*

\begin{tabular}{|c|c|c|}
\hline Case 17 & SP & NEP \\
\hline Pathogen identification & Acinetobacter baumannii & Acinetobacter baumannii \\
\hline Gentamicin & S & S \\
\hline Levofloxacin & S \\
\hline Aztreonam & I \\
\hline Piperacillin & S \\
\hline Piperacillin/ & S \\
\hline Tazobactam & S \\
\hline Cefepime & S & \\
\hline Ceftriaxone & I & \\
\hline Ceftazidime & S & \\
\hline Cefotetan & S & \\
\hline Tobramycin & S \\
\hline
\end{tabular}

S: Sensitive; R: Resistant; I: Intermediate; ${ }^{*}$ The kappa consistency test was used, Kappa value $=1.000$, indicating that the consistency of SP and NEP was excellent.

Table 5. Antibiotics susceptibility tests for Enterococcus faecium with two procedures*

\begin{tabular}{|c|c|c|}
\hline Case 18 & SP & NEP \\
\hline Pathogen identification & Enterococcus faecium & Enterococcus faecium \\
\hline Nitrofurantoin & $\mathrm{R}$ & $\mathrm{R}$ \\
\hline Erythroprim/sulfa & $\mathrm{R}$ & $\mathrm{R}$ \\
\hline Ciprofloxacin & $\mathrm{R}$ & \\
\hline Clindamycin & $\mathrm{R}$ & $\mathrm{R}$ \\
\hline Quinupristin/dalfopristin & $\mathrm{S}$ & $\mathrm{R}$ \\
\hline Moxifloxacin & $\mathrm{R}$ & $\mathrm{R}$ \\
\hline Tetracycline & $\mathrm{S}$ & \\
\hline Vancomycin & $\mathrm{S}$ & $\mathrm{R}$ \\
\hline Levofloxacin & $\mathrm{R}$ \\
\hline Benzylpenicillin & $\mathrm{R}$ \\
\hline Linezolid & $\mathrm{S}$ \\
\hline Ampicillin & $\mathrm{R}$ \\
\hline
\end{tabular}

S: Sensitive; R: Resistant; *The kappa consistency test was used, Kappa value $=1.000$, indicating that the consistency of SP and NEP was excellent. 
Table 6. Antibiotics susceptibility tests for Pseudomonas aeruginosa with two procedures*

\begin{tabular}{|c|c|c|}
\hline Case 19 & SP & NEP \\
\hline Pathogen identification & Pseudomonas aeruginosa & Pseudomonas aeruginosa \\
\hline ciprofloxacin & S & S \\
\hline Levofloxacin & S & S \\
\hline amikacin & S & S \\
\hline meropenem & S & S \\
\hline Piperacillin & $\mathrm{S}$ & $\mathrm{S}$ \\
\hline Piperacillin/ tazobactam & $\mathrm{S}$ & $\mathrm{S}$ \\
\hline gentamicin & $\mathrm{S}$ & $\mathrm{S}$ \\
\hline cefepime & $\mathrm{S}$ & $\mathrm{S}$ \\
\hline Ceftazidime & $\mathrm{R}$ & $\mathrm{S}$ \\
\hline Tobramycin & $\mathrm{S}$ & $\mathrm{S}$ \\
\hline Imipenem & $\mathrm{S}$ & $\mathrm{S}$ \\
\hline
\end{tabular}

S: Sensitive; R: Resistant; *The kappa consistency test was used, Kappa value $>0.9$, indicating that the consistency of SP and NEP was excellent.

Table 7. Antibiotics susceptibility tests for Enterobacter aerogenes with two procedures*

\begin{tabular}{|c|c|c|}
\hline Case 20 & SP & NEP \\
\hline Pathogen identification & Enterobacter aerogenes & Enterobacter aerogenes \\
\hline Amikacin & $\mathrm{S}$ & $\mathrm{S}$ \\
\hline Ampicillin/ sulbactam & $\mathrm{R}$ & $\mathrm{R}$ \\
\hline Nitrofurantoin & $\mathrm{I}$ & $\mathrm{I}$ \\
\hline Trimethoprim/ sulfa & $\mathrm{R}$ & $\mathrm{R}$ \\
\hline Ciprofloxacin & $\mathrm{S}$ & $\mathrm{S}$ \\
\hline Meropenem & $\mathrm{S}$ & $\mathrm{S}$ \\
\hline Piperacillin & $\mathrm{R}$ & $\mathrm{R}$ \\
\hline Piperacillin/ tazobactam & $\mathrm{I}$ & $\mathrm{S}$ \\
\hline Gentamicin & $\mathrm{S}$ & $\mathrm{S}$ \\
\hline Cefepime & $\mathrm{S}$ & $\mathrm{S}$ \\
\hline Cefuroxime & $\mathrm{R}$ & $\mathrm{R}$ \\
\hline Ceftriaxone & $\mathrm{R}$ & $\mathrm{R}$ \\
\hline Ceftazidime & $\mathrm{R}$ & $\mathrm{R}$ \\
\hline Tobramycin & $\mathrm{S}$ & $\mathrm{S}$ \\
\hline Imipenem & $\mathrm{I}$ & $\mathrm{S}$ \\
\hline Levofloxacin & $\mathrm{S}$ & $\mathrm{S}$ \\
\hline Cefazolin & $\mathrm{R}$ & $\mathrm{R}$ \\
\hline
\end{tabular}

S: Sensitive; R: Resistant; I: Intermediate; *The kappa consistency test was used, Kappa value $=0.805,95 \%$ CI: $0.564-1.000$, Kappa $>0.9$, indicating that the consistency of SP and NEP was excellent.

Again, this simple omitted one step modification is based on the facts that: (A) in most of cases, the invaded pathogen that causes sepsis is only single strain; (B) the SP does not separate colonies but collects all colonies from overnight cultured-dish, which allows us to omit this re-culture step and directly take $10 \mathrm{ml}$ positive media to enrich the pathogen for PI and AST. Of cause, we do not against the day 2 dish culture for other purposes; such as further explore the other biological properties of pathogen. Since there are at least $20 \mathrm{ml}$ media plus blood in the BD BACTEC bottle, taken $10 \mathrm{ml}$ to perform an early diagnosis for the urgent needs of clinical medical decision is practicable and should be of benefit to patients.

Indeed, Accelerate Pheno" System and Accelerate PhenoTest" BC Kit, a newly FDA cleared fast diagnostic testing system (Accelerate Diagnostics Inc, 3950 S. Country Club Road, Tucson, Arizona, USA 85714) uses the same concept of one strain invaded to cause sepsis and directly takes microbes in positive blood culture bottle for PI and AST. As claimed by Accelerate Diagnostics Inc, their fully automated new device for AST could be less work, less workflow, at least $40 \mathrm{~h}$ fast to yield report, less patient wait and shorter hospital stay (http:// acceleratediagnostics.com). The use of Accelerate Pheno" System provides a promising data in clinical setting $[17,19]$. The results of AST of direct use of broth from positive blood cultures and inoculated reculture dish were also compared among the BacT/ALERT, BACTEC and VersaTREK systems, and the categorical agreement analysis was also very promising [18]. Increasing efforts are focused on direct use of broth of positive culture instead of re-inoculation to dish to obtain the isolates. Along this line, while several automation devices have been developed [19-20], the price of devices is relatively high, so that most of the hospitals or laboratories are reluctant to use the new devices and reagents with 1-2 delaying PI and AST reports [12]. Our NEP is an easy and economical alternative approach to achieve at least one day earlier report as newly developed devices without changing currently used device and reagents. By omitted one step, NEP is more cost-effective. Once the automation devices become cheaper and yield more advantages with FDA clearance, then, it can be shifted to use the advanced devices and reagents.

The bottle neck for this NEP is that the $10 \mathrm{ml}$ culture media should be cleaned up both blood and other media portion as much as possible for not to interfere the following PI and AST assays. Several cautions need to be taken: (1) completely lyse the blood cells, since the bio-properties of cell plasma membrane and bacterial wall are quite different, the lysis time can be up to 12-18 min to ensure the blood cells are all lysed; (2) use sterilized saline or water to spin -washout the bacterial pellets; (3) collect only white pellets mainly with bacterial and justify to $0.5 \mathrm{McF}$ to fit the assay needs; and (4) avoid any contamination in reagents and materials used during the operation process.

Although it is a simple modification of omitted one step without changing current device and reagent setting in any hospital, it really yields a great benefit of rescuing the sepsis patients by giving one day in advance for physicians to make a right antibiotics selection.

With the emergence of antibiotic-resistant bacteria as an increasing cause of sepsis death [21-22], we need to pay more attention not only in the fast reporting AST, but also the assays that detect the antibioticresistant bacteria. More studies need to be carried out to confirm the NEP efficacy and develop new assays for antibiotic-resistant bacteria.

\section{Acknowledgements}

This study was supported in part by grants from: International Science \& Technology Cooperation Program of China (2015DFA31770) to JL; Fujian Development and Reform Commission to JL (FGW2014). We thank Dr. Shimin Zhang for editing this manuscript for publication.

\section{Conflicts of interest}

Authors declare no duality of interest.

\section{References}

1. Schuts EC, Hulscher MEJL, Mouton JW, Verduin CM, Stuart JWTC, et al. (2016) Current evidence on hospital antimicrobial stewardship objectives: a systematic review and meta-analysis. Lancet Infect Dis 16: 847-856. [Crossref]

2. Pfafflin A, Schleicher E (2009) Inflammation markers in point-of-care testing (POCT) Anal Bioanal Chem 297: 1473-1480. [Crossref]

3. Iregui M, Ward S, Sherman G, Fraser VJ, Kollef MH (2002) Clinical importance of delays in the initiation of appropriate antibiotic treatment for ventilator-associated pneumonia. Chest 122: 262-268. [Crossref]

4. Luna CM, Aruj P, Niederman MS, Garzón J, Violi D, et al. (2006) Appropriateness and delay to initiate therapy in ventilator-associated pneumonia. Eur Respir J27:158-164. [Crossref]

5. Kibe S, Adams K, Barlow G (2011) Diagnostic and prognostic biomarkers of sepsis in critical care. J Antimicrob Chemother 66 Suppl 2: ii33-40. [Crossref]

6. Barenfanger J, Drake C, Kacich G (1999) Clinical and financial benefits of rapid bacterial identification and antimicrobial susceptibility testing. J Clin Microbiol 37 1415-1418. [Crossref] 
7. Doern GV, Vautour R, Gaudet M, Levy B (1994) Clinical impact of rapid in vitro susceptibility testing and bacterial identification. JClin Microbiol 32: 1757-1762. [Crossref]

8. Turner KM, Christensen H, Adams EJ, McAdams D, Fifer H, et al. (2017) Analysis of the potential for point-of-care test to enable individualised treatment of infections caused by antimicrobial-resistant and susceptible strains of Neisseria gonorrhoeae: a modelling study. BMJ Open 6: e015447. [Crossref]

9. Beuving J, van der Donk CF, Linssen CF, Wolffs PF, Verbon A (2011) Evaluation of direct inoculation of the BD PHOENIX system from positive BACTEC blood cultures for both Gram-positive cocci and Gram-negative rods. BMC Microbiol 30: 156. [Crossref]

10. Granato PA (1993) The impact of same-day tests versus traditional overnight testing. Diagn Microbiol Infect Dis 16: 237-243. [Crossref]

11. Chandrasekaran S, Abbott A, Campeau S, Zimmer BL, Weinstein M, et al. (2018) Direct-from-blood culture disk diffusion to determine antimicrobial susceptibility of Gram-negative bacteria: Preliminary report from the Clinical and Laboratory Standards Institute Methods Development and Standardization Working Group. J Clin Microbiol pii: JCM.01678-17. [Crossref]

12. Tabak YP, Vankeepuram L, Ye G, Jeffers K, Gupta V, et al. (2018) Blood Culture Turnaround Time in US Acute Care Hospitals and Implications for Laboratory Process Optimization. J Clin Microbiol pii: JCM.00500-18. [Crossref]

13. Pulido MR, García-Quintanilla M, Martín-Peña R, Cisneros JM, McConnell MJ (2013) Progress on the development of rapid methods for antimicrobial susceptibility testing. J Antimicrob Chemother 68: 2710-2717. [Crossref]

14. Murray MT, Johnson CL, Cohen B, Jackson O, Jones LK, et al. (2018) Use of antibiotics in paediatric long-term care facilities. J Hosp Infec 99: 139-144. [Crossref]
15. Bauer M, Reinhart K (2010) Molecular diagnostics of sepsis--where are we today? Int J Med Microbiol 300: 411-413. [Crossref]

16. American College of Chest Physicians/Society of Critical Care Medicine Consensus Conference (1992) Definitions for sepsis and organ failure and guidelines for the use of innovative therapies in sepsis. Crit care med 20: 864-874. [Crossref]

17. Greenhalgh DG, Saffle JR, Holmes JH 4th, Gamelli RL, Palmieri TL, et al. (2007) American Burn Association consensus conference to define sepsis and infection in burns. J Burn Care Res 28: 776-790. [Crossref]

18. Hanisch E, Brause R, Paetz J, Arlt B (2011) Review of a large clinical series: Predicting death for patients with abdominal septic shock. J Intensive Care Med 26: 27-33. [Crossref]

19. Marschal M, Bachmaier J, Autenrieth I, Oberhettinger P, Willmann M, Peter S (2017) Evaluation of the Accelerate Pheno System for Fast Identification and Antimicrobial Susceptibility Testing from Positive Blood Cultures in Bloodstream Infections Caused by Gram-Negative Pathogens. J Clin Microbiol 55: 2116-2126. [Crossref]

20. Chandrasekaran S, Abbott A, Campeau S, Zimmer BL, Weinstein M, et al. (2018) Direct-from-blood culture disk diffusion to determine antimicrobial susceptibility of Gram-negative bacteria: Preliminary report from the Clinical and Laboratory Standards Institute Methods Development and Standardization Working Group. J Clin Microbiol pii: JCM.01678-17. [Crossref]

21. Ersoy SC, Heithoff DM, Barnes L, Tripp GK, House JK, et al. (2017) Correcting a Fundamental Flaw in the Paradigm for Antimicrobial Susceptibility Testing. EBioMedicine 20: 173-181. [Crossref]

22. Kaushik AM, Hsieh K, Chen L, Shin DJ, Liao JC, et al. (2017) Accelerating bacteria growth detection and antimicrobial susceptibility assessment in integrated picoliter droplet platform. Biosens Bioelectron 97: 260-266. [Crossref]

Copyright: (C2018 Lin J. This is an open-access article distributed under the terms of the Creative Commons Attribution License, which permits unrestricted use, distribution, and reproduction in any medium, provided the original author and source are credited. 